\title{
Quality assessment: Qualitative and quantitative analysis of nine active components in Sargentodoxa cuneata by HPLC/Q Exactive Plus MS and UPLC-ESI- MS/MS
}

\section{Lin Zheng}

Guizhou Medical University

Zu-Ying Zhou

Guizhou Medical University

Chang-Quan Wang

Guizhou Medical University

\section{Yong-Lin Wang}

Guizhou Medical University

\section{Zi-Peng Gong}

Guizhou Medical University

\section{Yue-Ting Li}

Guizhou Medical University

\section{Ai-Min Wang}

Guizhou Medical University

\section{Si-Ying Chen}

Guizhou Medical University

\section{Xue Ma}

Guizhou Medical University

\section{Yong-Jun Li}

Guizhou Medical University

Yong Huang ( $\sim$ HUANGY2020@126.com )

Guizhou Medical University

\section{Research}

Keywords: Sargentodoxa cuneata, hierarchical clustering analysis, principal components analysis, partial least squares discriminant analysis, quality assessment, HPLC/Q Exactive Plus MS, UPLC-ESI-MS/MS

Posted Date: July 27th, 2020

DOl: https://doi.org/10.21203/rs.3.rs-45959/v1 
License: (a) (i) This work is licensed under a Creative Commons Attribution 4.0 International License. Read Full License 


\section{Abstract}

Background: Sargentodoxa cuneata is an herb commonly used by the Hmong people, and many preparations containing this herb have been marketed. However, the source of medicinal materials is not standardized during the production process, and the quality of medicinal materials from different sources may be inconsistent. This study was aimed to establish a qualitative and quantitative analysis method for quality assessment of Sargentodoxa cuneata in different regions.

Methods: HPLC/Q Exactive Plus MS was performed in positive mode and negative mode used for qualitative analysis, and there were twenty-one ingredients were tested. Nine high-content or characteristic ingredients were screened out and applied to the quantitative analysis of thirty batches of medicinal materials from different origins. Based on the results of the quantitative analysis, further multivariate statistical analyses were carried out for quality assessment of Sargentodoxa cuneata .

Results: Results showed that there were significant differences in the contents of the nine ingredients of Sargentodoxa cuneata in the eastern and southwestern regions. The content of hydroxytyrosol, salidroside, chlorogenic acid, desrhamnosyl isoacteoside, and liriodendrin of Sargentodoxa cuneata in eastern region was higher, while the content of protocatechin in southwestern region was higher. The different ingredients included protocatechuic acid, desrhamnosyl isoacteoside, liriodendrin, and hydroxytyrosol. These four ingredients were important factors that caused the difference in the quality of Sargentodoxa cuneata from different origins.

Conclusion: Protocatechuic acid, desrhamnosyl isoacteoside, liriodendrin, and hydroxytyrosol may become a new marker for quality assessment of Sargentodoxa cuneata.

\section{Background}

Sargentodoxa cuneata (DXT), also called "DaHuoxue", "HongPiTeng", "HongTeng", etc., was the dried cane of Largentizabalaceae plant Sargentodoxa cuneata (Oliw.) Rehd. Et Wils., which was first published in "WaiMuMan" class of "Ben Cao Tu Jing. Ben Jing" [1]. It was harvested in the autumn and winter, with side branches removed, cut and dried. And its taste was bitter, and medicinal properties were flat, attributed to the large intestine and liver meridian. It had the effects of detoxification, promoting blood circulation, removing wind and pain, used for intestinal carbuncle abdominal pain, heat poisoning sores, amenorrhea, dysmenorrhea, tumbling pain and rheumatic arthralgia, which was mainly produced in Anhui, Guizhou, Guangxi, Sichuan, Yunnan, Hubei, and other provinces [2]. DXT was also a traditional medicinal material for the Miao people, which belonged to the "blood circulation" of the "four major blood" of the Miao medicine. DXT was commonly used by Miao nationality to promote blood circulation, remove wind and dehumidify, as well as the treatment of rheumatic bone pain and bruises [3]. The preparations of Compound Xueteng Yaojiu, Guyanning granules, Miao Yaoshengxian decoction, Tongqiaohua suppository decoction, etc. all contained DXT medicinal materials, which had good curative effect [4-6]. Modern pharmacological research [7-11] also showed that DXT and its extracts had various physiological activities such as analgesic, anti-inflammatory, antibacterial, anti-oxidation, anti-viral and so on. 
Current standards and works only required identification of its traits, microscopic characteristics [1], moisture, total ash, leachables [2], and thin-layer chromatography [12] to control the quality of medicinal materials, and the requirements for the specific chemical component contained in medicinal materials were not clear. In the published literature, only salidroside $[13,14]$, chlorogenic acid $[9,13,15]$, total flavonoids $[16$, 17], proanthocyanidins [18], volatile oil [19], emodin [20], protocatechuic acid [15], or a few or a class of components were measured. The DXT production areas were widely distributed, and the content of chemical components in different production areas was also different. In the reports of multi-batch multi-component content measurement, Li Hao [21] used spectrophotometry and HPLC to determine the content of total phenols, total saponins, and index components of salidroside, chlorogenic acid, and 3,4dihydroxyphenylethanol glucoside. Li Dihua [22] used HPLC coupled with evaporative light scattering detection to simultaneously determine the content of four active ingredients (3,4-dihydroxyphenylethyl alcohol glycoside, salidroside, chlorogenic acid, and liriodendrin) in thirteen test samples. However, the resolution and sensitivity of these measurement methods were not very high, and the measurement results had certain limitations.

Therefore, in this study, an accurate, efficient, and convenient method for the determination of multiple batches of DXT in different regions was established. HPLC tandem Q Exactive Plus MS was performed to full-component qualitative analysis and the higher-content or characteristic components were screened out. Subsequently, the contents of the selected components were determined using UPLC-ESI-MS/MS. According to the measured data, further multivariate statistical analysis was carried out to find the differences in the content of the selected components in DXT in different regions. This might provide a certain basis for improving DXT quality control standards.

\section{Materials And Methods}

\section{Chemicals and reagents}

The chemical standards of chlorogenic acid (110753-201817) and protocatechuic acid (111538-201606) were obtained from National Institutes for Food and Drug Control (Beijing, China). The standard substances of caffeic acid (AF9051082), rutin (AF8032520), epicatechin (AF8030805), desrhamnosyl isoacteoside (AF9102921), liriodendrin (AF9102701), hydroxytyrosol (AF8040805) were purchased from Chengdu Alfa Biotech Co. Ltd (Chengdu, China). And the reference substance of salidroside (160820) was supplied from Beijing Century Biotech Co., Ltd. (Beijing, China). The purity of each compound was $>98 \%$, and other reagents were of analytical grade.

\section{Plant materials}

DXT samples were collected from different provinces in China. These herbs were identified by Professor Long Qingde (Pharmacy, School of Pharmacy, Guizhou Medical University). The voucher specimen was deposited in the herbarium of Guizhou Medical University School of Pharmacy.

\section{Preparation of standard solutions}


Precisely weighed the appropriate amount of the reference substances and diluted to $10 \mathrm{~mL}$ with methanol, and initial stock solutions of chlorogenic acid $(1.02 \mathrm{~g} / \mathrm{L})$, protocatechuic acid $(1.035 \mathrm{~g} / \mathrm{L})$, caffeic acid $(0.502 \mathrm{~g} / \mathrm{L})$, rutin $(0.508 \mathrm{~g} / \mathrm{L})$, epicatechin $(1.018 \mathrm{~g} / \mathrm{L})$, desrhamnosyl isoacteoside $(1.024 \mathrm{~g} / \mathrm{L})$, liriodendrin

$(0.760 \mathrm{~g} / \mathrm{L})$, hydroxytyrosol $(1.998 \mathrm{~g} / \mathrm{L})$ and salidroside $(1.020 \mathrm{~g} / \mathrm{L})$ were obtained. Accurately measured and diluted the above 9 initial stock solutions to obtain the final concentration stock solutions of chlorogenic acid $(0.255 \mathrm{~g} / \mathrm{L})$, protocatechuic acid $(0.015 \mathrm{~g} / \mathrm{L})$, and caffeic acid $(0.015 \mathrm{~g} / \mathrm{L})$, rutin $(0.008 \mathrm{~g} / \mathrm{L})$, epicatechin $(0.061 \mathrm{~g} / \mathrm{L})$, desrhamnosyl isoacteoside $(0.015 \mathrm{~g} / \mathrm{L})$, liriodendrin $(0.060 \mathrm{~g} / \mathrm{L})$, hydroxytyrosol $(0.029 \mathrm{~g} / \mathrm{L})$ and salidroside $(0.046 \mathrm{~g} / \mathrm{L})$. Accurately measured and mixd $100 \mu \mathrm{L}$ of the nine final concentration stock solutions respectively to obtain a mixed series of standard solutions, which were sequentially diluted by a 2fold dilution method and stored in a refrigerator $\left(-20^{\circ} \mathrm{C}\right)$ for future use.

\section{Preparation of sample solutions}

Weighed $0.2 \mathrm{~g}$ of DXT medicinal material, added $25 \mathrm{~mL}$ of $50 \%$ methanol, weighed and refluxed for $2 \mathrm{~h}$, added $50 \%$ methanol to make up the weight, took $200 \mu \mathrm{L}$, added $50 \%$ methanol $800 \mu \mathrm{L}$, vortexed, centrifuged at 12, $000 \mathrm{rpm}, 10 \mathrm{~min}$. The supernatant sample was taken to inject, and the injection volume was $1 \mu \mathrm{L}$. The final concentration of the DXT extract was $1.0 \mathrm{~g} / \mathrm{mL}$.

\section{Results}

\section{Identification of constituents in the samples by HPLC and Ultra-high-resolution MS}

Vanquish horizon HPLC system combined with Q Exactive Plus ultra-high resolution mass spectrometry (Thermo Fisher Scientific, MA, USA) and compound discovery structure identification software was used to detect the main chemical components in DXT extraction solution samples. The precursor ion selectivity of the conjugate hyperboloid quadrupole combined with the high resolution accurate mass detection technology based on orbitrap had the advantages of ultra-high resolution $(R=140,000 @ \mathrm{~m} / \mathrm{z} 200$, could quickly switch between positive and negative at the same time to obtain comprehensive sample information), ultra-high quality accuracy and stability, high sensitivity (ag-fg level) and ultra-wide linear range $\left(10^{4}-10^{5}\right)$, which could greatly increase the credibility and efficiency of identification.

The chromatographic conditions were as follows: Column, Hypersil gold aQ $(2.1 * 100 \mathrm{~mm}$, $1.9 \mu \mathrm{m}$ ); Mobile phase, $0.04 \%$ acetic acid water (A) - 0.04\% acetonitrile acetate (B); Flow rate, $0.35 \mathrm{~mL} / \mathrm{min}$; The injection volume, $2 \mu \mathrm{L}$. The elution gradient was shown in Additional file 1. 
In the Q Exactive Plus MS system, an electrospray ion source was used for detection in the positive and negative ion scanning modes, and the conditions are as follows: Spray voltage: $3.8 \mathrm{kV}(+) / 3.2 \mathrm{kV}(-)$; Vaporiser temp: $350{ }^{\circ} \mathrm{C}$; Sheath gas: 40 arb; AUX gas: 10 arb; Capillary Temp: $320^{\circ} \mathrm{C} \rrbracket$ s-Lens: 60; General Method: Fullms-ddms2₫Scan range: 120-1500₫Resolution (MS1): 70, 000; MS/MS resolution: 17, 500; Stepped NCE: 20, 40, 60.

The total ion chromatograms in negative ion and positive ion modes were shown in Fig.1 and 2 , and the mass spectrum details of the measured component obtained from the detector were shown in Table 1. A total of twenty-one components were tested and identified. Based on preliminary experiments, nine components with high activity or high content were selected for subsequent content determination. The nine ingredients were protocatechuic acid, hydroxytyrosol, caffeic acid, epicatechin, salidroside, chlorogenic acid, desrhamnosyl isoacteoside, rutin, and liriodendrin. And the chemical formulas were shown in Additional file 2 .

Table 1 Mass spectrum information of tested components 


\begin{tabular}{|c|c|c|c|c|}
\hline Detection mode & Peak & Retention time / $t_{R}(\min )$ & Excimer ion & Component attribution \\
\hline Negative mode & $\mathrm{a}$ & 3.41 & 167.03 & Vanillic acid \\
\hline \multirow{17}{*}[\mathrm{M}-\mathrm{H}]{$^{-}(\mathrm{M} / \mathrm{Z})$} & $\mathrm{b}$ & 3.52 & 153.01 & Protocatechuic acid \\
\hline & $\mathrm{c}$ & 3.96 & 153.05 & Hydroxytyrosol \\
\hline & $\mathrm{d}$ & 4.38 & 299.11 & Salidroside \\
\hline & e & 4.63 & 353.08 & Chlorogenic acid \\
\hline & $\mathrm{f}$ & 4.81 & 137.02 & $p$-hydroxybenzoic acid \\
\hline & g & 5.36 & 179.03 & Caffeic acid \\
\hline & $\mathrm{h}$ & 5.48 & 289.07 & Epicatechin \\
\hline & $\mathrm{i}$ & 6.67 & 477.14 & Desrhamnosyl isoacteoside \\
\hline & j & 8.00 & 193.05 & Ferulic acid \\
\hline & $\mathrm{k}$ & 8.29 & 312.12 & Feruloyltyramine \\
\hline & 1 & 12.20 & 487.34 & Madasiatic acid \\
\hline & $\mathrm{m}$ & 5.17 & 577.16 & Procyanidin B2 \\
\hline & $\mathrm{n}$ & 5.86 & 741.71 & Liriodendrin \\
\hline & o & 8.67 & 283.06 & Physcion \\
\hline & $\mathrm{p}$ & 8.73 & 609.14 & Rutin \\
\hline & q & 9.55 & 685.38 & Rosamultin \\
\hline & $\mathrm{r}$ & 17.79 & 17.79 & Oleanolic acid \\
\hline Positive mode & $\mathrm{a}$ & 2.03 & 167.07 & Apocynin \\
\hline \multirow{3}{*}[\mathrm{M}+\mathrm{H}]{$^{+}(\mathrm{M} / \mathrm{Z})$} & $\mathrm{b}$ & 5.48 & 291.07 & Epicatechin \\
\hline & c & 5.68 & 271.05 & Emodin \\
\hline & $d$ & 6.61 & 303.04 & Quercetin \\
\hline
\end{tabular}

\section{Method Validation}

The specificity, calibration curves, limits of detection (LOD), quantitation (LOQ), precision, stability, repeatability, and recovery were investigated. 
The specificity was investigated by taking the mixed reference solution and $50 \%$ methanol-water blank solvent, and performing sample analysis according to the optimized LC/MS conditions. The calibration curve for each constituent was established by plotting the peak area $(y)$ versus the concentration $(x)$ of each analyte. The LOD and LOQ for nine analytes were estimated at S/N of 3 and 10, respectively, by injecting a series of dilute solutions with known concentration. The intra-day and inter-day precision for each analyte was investigated by determining the nine analytes in six replicates during a single day and three consecutive days. Variations of the peak area were taken as the measures of intra-day and inter-day analysis precision to calculate the RSD. Stability was investigated by analyzing the solution at $0,2,4,8,12,24 \mathrm{~h}$, respectively. To assess the repeatability, six solutions prepared from sample were analyzed. Variability was expressed in RSD (\%). The recoveries of the analytes were determined by using the method of standard addition within the same day. Three different concentrations of mixed standard solutions (50\%, 100\%, and $150 \%$ of the known amount in sample) were spiked into sample. The recovery results were calculated by comparing the difference between the spiked and the un-spiked sample that were analyzed under the same conditions.

The extracted ion chromatogram obtained from the specificity validation was shown in Fig.3, indicating that the method had good specificity. The calibration curve had good linearity, and LOD and LOQ also met the requirements (as shown in Table 2). During the investigation of the precision, stability, repeatability and sample recovery rate of the nine components, the RSD values were all $\leq 3.2 \%$, which met the requirements. These results were shown in Additional file 3, which showed that the method was stable and reliable.

Table 2 Calibration curve, LOD, and LOQ 


\begin{tabular}{cccccc}
\hline Analytes & $y=\mathrm{Ax}+\mathrm{B}$ & Linear range & $\boldsymbol{R}^{2}$ & LOD & LOQ \\
& & $(\mu \mathrm{g} / \mathrm{mL})$ & & $(\mu \mathrm{g} / \mathrm{mL})$ & $(\boldsymbol{\mu g} / \mathrm{mL})$ \\
\hline Protocatechuic acid & $y=244691 x-2895.5$ & $1.553-0.025$ & 0.9991 & 0.0036 & 0.012 \\
Hydroxytyrosol & $y=20152 x-635.69$ & $2.997-0.047$ & 0.9997 & 0.0050 & 0.015 \\
Caffeic acid & $y=534896 x-6034.8$ & $0.753-0.012$ & 0.9997 & 0.0026 & 0.006 \\
Epicatechin & $y=28186 x+766.44$ & $6.108-0.096$ & 0.9992 & 0.0080 & 0.024 \\
Salidroside & $y=30434 x-183.7$ & $2.295-0.036$ & 0.9990 & 0.0030 & 0.018 \\
Chlorogenic acid & $y=217299 x-29966$ & $25.500-0.398$ & 0.9994 & 0.1250 & 0.025 \\
Desrhamnosyl isoacteoside & $y=122310 x-2488.7$ & $1.536-0.024$ & 0.9991 & 0.0030 & 0.010 \\
Rutin & $y=9600.2 x-131.34$ & $0.813-0.013$ & 0.9988 & 0.0026 & 0.006 \\
Liriodendrin & $y=13136 x-1540.9$ & $6.004-0.094-$ & 0.9973 & 0.0060 & 0.023 \\
\hline
\end{tabular}

\section{Apparatus and optimal chromatographic and MS/MS conditions}

An ACQUITY I-Class UPLC system (Waters Corp, MA, USA) with a conditional auto-sampler and an Acquity I-Class UPLC BEH C18 Column (2.1 mm × $100 \mathrm{~mm}$, internal diameter $1.7 \mu \mathrm{m})$ was used for the analyses. The system was also equipped with a Waters VanGuard BEH C18 (2.1 $\mathrm{mm} \times 5 \mathrm{~mm}, 1.7 \mu \mathrm{m})$ column. The column and auto-sampler were maintained at $40{ }^{\circ} \mathrm{C}$ and $25^{\circ} \mathrm{C}$, respectively. The injection volume was $1 \mu \mathrm{L}$. The gradient mobile phase system consisting of $0.1 \%$ formic acid in acetonitrile (B) and $0.1 \%$ aqueous formic acid (A) was applied at a flow rate of $0.3 \mathrm{~mL} / \mathrm{min}$ and run time of $6 \mathrm{~min}$. The mobile phase consisted of $0.1 \%$ formic acid in water as solvent $\mathrm{A}$ and $0.1 \%$ formic acid in acetonitrile as solvent $\mathrm{B}$. The gradient program was as follows: 0 - $0.5 \mathrm{~min}, 10 \%$ 10\% B; $0.5-2.5$ min linear gradient 10\% $\sim 40 \%$ B; $2.5-4 \min , 40 \% \sim 90 \%$ B; $4-4.5 \min , 90 \% \sim 90 \%$ B; $4.5-6 \min , 90 \% \sim 10 \%$ B. The flow rate was $0.3 \mathrm{~mL} / \mathrm{min}$. The injection volume was $1 \mu \mathrm{L}$ with partil loop mode. The temperature of the sample manager was maintained at $15{ }^{\circ} \mathrm{C}$.

Mass spectrometric detection was performed with an XEVO TQS Triple-Quadrupole Tandem Mass Spectrometer (Waters Corp, Milford, MA, USA) equipped with an electrospray ionization (ESI) source. The mass spectrometer parameters were: Capillary voltage, $3.0 \mathrm{kV}$; 
Capillary ionization voltage, $3 \mathrm{kV}$; ion source temperature, $120^{\circ} \mathrm{C}$; spray gas and backflush gas, $\mathrm{N}_{2}$; desolvation gas flow rate, $650 \mathrm{~L} / \mathrm{h}$; and desolvation gas temperature, $350{ }^{\circ} \mathrm{C}$.

Multiple reaction monitoring (MRM) mode was used for quantification. The optimal parameters for the analytes in the MRM mode are listed in Table 3. All data were obtained using MasslynxTM V4.1 software and processed using the QuanlynaTM V4.1 (Waters Corp., Millford, MA, USA) workstation.

Table 3 Mass spectrometric parameters of nine determination components

\begin{tabular}{|c|c|c|c|c|c|}
\hline Component & Molecular formula & Retention time $/ t_{R}(\min )$ & Quantitative ion pair & Cone voltage & Collision energy \\
\hline Protocatechuic acid & $\mathrm{C}_{7} \mathrm{H}_{6} \mathrm{O}_{4}$ & 0.89 & $152.9-109$ & 30 & 15 \\
\hline Hydroxytyrosol & $\mathrm{C}_{8} \mathrm{H}_{10} \mathrm{O}_{3}$ & 0.90 & $153.5-123$ & 20 & 15 \\
\hline Caffeic acid & $\mathrm{C}_{9} \mathrm{H}_{8} \mathrm{O}_{4}$ & 1.50 & $178.8-134.8$ & 35 & 15 \\
\hline Epicatechin & $\mathrm{C}_{15} \mathrm{H}_{14} \mathrm{O}_{6}$ & 1.57 & $289.1-244.6$ & 35 & 15 \\
\hline Salidroside & $\mathrm{C}_{14} \mathrm{H}_{20} \mathrm{O}_{7}$ & 0.95 & 299.1-119 & 35 & 20 \\
\hline Chlorogenic acid & $\mathrm{C}_{16} \mathrm{H}_{18} \mathrm{O}_{9}$ & 1.24 & 353-191 & 30 & 15 \\
\hline Desrhamnosyl isoacteoside & $\mathrm{C}_{23} \mathrm{H}_{26} \mathrm{O}_{11}$ & 2.05 & $477-161$ & 30 & 15 \\
\hline Rutin & $\mathrm{C}_{27} \mathrm{H}_{30} \mathrm{O}_{16}$ & 1.91 & $609.2-300$ & 30 & 15 \\
\hline Liriodendrin & $\mathrm{C}_{34} \mathrm{H}_{46} \mathrm{O}_{18}$ & 1.81 & 741.7-417.5 & 30 & 25 \\
\hline
\end{tabular}

\section{Determination of UPLC-ESI-TQS MS/MS content of nine components in thirty batches of DXT}

Nine components with higher content or stronger activity from the tested components were selected, and UPLCESI-TQS MS/MS was used to determine the content of different batches of DXT medicinal materials in thirty different regions under the above optimized conditions. The content determination results were shown in Table 4 .

\section{Quality assessment}

Hierarchical clustering analysis 
In order to analyze the changes in the content of each component between DXT medicinal materials in different regions as a whole, SPSS software (IBM SPSS Statistics 20.0 Developer, IBM Corp., NY, USA) was used to perform hierarchical clustering analysis (HCA) on the content determination data of nine components in thirty batches of DXT medicinal materials. Using the ward method, the squared Euclidean distance was selected as the metric to perform HCA. The HCA dendrogram was shown in Fig.4. It could be seen that thirty samples were divided into two major clusters (I and II) based on the contents of nine compounds. Samples from Guizhou, Sichuan, Yunnan, and Guangxi were included in the cluster I, while samples from eastern provinces such as Anhui, Hubei, and Jiangxi were clustered into the cluster II, which reflected the similar content of chemical composition in the same cluster of samples. In addition, it was also found that the areas of cluster I was located in the southwest of China, while the area of cluster II belongs to east of China. Obviously, the differences between the east and the southwest of China affect the components of DXT, such as geographic location, climate, and precipitation [23].

Cluster I was further divided into another two branches (group $\square$ and $\square$ ). It could be found that all samples from Sichuan, and Bijie and Zunyi of Guizhou were grouped into group [, while samples from Guangxi and Yunnan, as well as Anshun, Duyun, Xingyi, Liupanshui of Guizhou were gathered in the group $\square$. Geographically, Bijie and Zunyi are adjacent to Sichuan, while Anshun, Duyun, Xingyi, Liupanshui is adjacent to Yunnan and Guangxi. And Guiyang is located in the central part of Guizhou, with similar distances from Sichuan, Yunnan, and Guangxi. In the dendrogram, samples from Guiyang were distributed in both group $\square$ and $\square$, which could explain to a certain extent that the quality of the DXT in southwest of China was not completely consistent.

\section{Principal components analysis}

In order to analyze the relationship between the content of the component and the region, principal components analysis (PCA) was performed using SPSS software (IBM SPSS Statistics 20.0 Developer, IBM Corp., NY, USA). And 2D and 3D plots of loading plot and score plot were obtained as shown in Fig.5.

The loading plot was used to describe data features, it could be known that the relationship between variable attribute features. And the distinction between samples could be got from the score plot. It can be seen from the calculation of the principal component (PC) that the larger the absolute value of the loading, the greater the influence on the PC. Three PCs were extracted (PC 1, 2 and 3), and the cumulative contribution rate was $77.18 \%$.

Combining the distribution characteristics (Additional file 4) and the loading plot (Fig.5 (a) and (b)) of the content, in the PC1 axis, protocatechuic acid and rutin were negatively correlated and other components were 
positively correlated. The contents of the first two component variables in the southwestern samples were higher than that in the eastern samples, while other components in the southwestern samples were less than that in eastern samples, which indicated that the differences in the contents of the component variables in different regions were mainly reflected in this PC. Additionally, hydroxytyrosol and desrhamnosyl isoacteoside were mainly moved on PC1 axis, while not obvious on other axis, indicating that these two components were almost completely affected by PC1, that is, the content of the two components in the eastern region was significantly higher than that in the western region, which was consistent with the quantitative results.

As shown in score plots (Fig.5 (c) and (d)), the samples in the eastern and southwest regions except sample 19 were almost completely separated, which was approximately consistent with HCA result. On the 2D graph, sample 25 from Guizhou Bijie was moved in the second quadrant, and other samples were distributed near by the PC1 axis. Additionally, the samples derived from eastern regions were shifted in the positive direction of PC1 axis, while the samples in the southwest region were mainly distributed in the negative direction of the axis. Analyzed with 2D load plot, it could be speculated that the medicinal materials from eastern region were mainly affected by the component variables desrhamnosyl isoacteoside, while the medicinal materials from southwestern region were mainly affected by the component variable protocatechuic acid. When the third PC was added, the sample 4 from Jiangxi Jiujiang moved significantly. The quantitative results showed that desrhamnosyl isoacteoside was significantly less than other samples in the same region, while the content of salidroside was significantly higher. Combining the results of loading plots and score plots, the main variable that affects sample 25 was rutin, and the main variable that made sample 4 moved was salidroside.

To sum up, the components that made the quality difference of DXT medicinal materials of the east and the southwest might include desrhamnosyl isoacteoside and protocatechuic acid.

\section{Partial least squares discriminant analysis}

In order to further screen the key components that caused the differences in the quality of DXT from various regions, the raw data of the nine measured components of DXT from different regions was input as variable values into SIMCA 14.1 software (Umetrics, Sweden) for partial least squares discriminant analysis (PLS-DA), the variable importance of projection (VIP) value of each index component was obtained. As shown in Fig.6, when VIP > 1 as the screening criterion, it could be concluded that protocatechuic acid, desrhamnosyl isoacteoside, liriodendrin, and hydroxytyrosol had significant differences in the DXT from different regions. 


\section{Discussion}

The extraction method (reflux method and ultrasonic method), solvent (various concentrations of methanol) and extraction time were studied to optimize the extraction procedure. The results were shown that the ultrasonic method could extract chemical components more effectively than the reflux method. Compared different extraction solvent conditions ( $25 \%, 50 \%, 75 \%$, and $100 \%$ methanol), $50 \%$ methanol showed complete extraction of all major components. When the pharmaceutical powder was extracted with $50 \%$ methanol under reflux for $0.5,1,2$, and $4 \mathrm{~h}$, reflux was obtained for $2 \mathrm{~h}$ to obtain the most complete extraction (Since there was no significant difference between 2 and $4 \mathrm{~h}, 2 \mathrm{~h}$ was sufficient for extraction.). Therefore, the preferred extraction method was refluxing in $50 \%$ methanol for 2 hours.

\section{Conclusion}

Protocatechuic acid, desrhamnosyl isoacteoside, liriodendrin, and hydroxytyrosol were important factors that caused the difference in the quality of Sargentodoxa cuneata from different origins, which may become a new marker for quality assessment of Sargentodoxa cuneata.

\section{Abbreviations}

UPLC-ESI-MS/MS: Ultra-performance liquid chromatography coupled with electrospray ionization- triple quadrupole system tandem mass spectrometry; DXT: Sargentodoxa cuneata; LOD: Limit of detection; LOQ: Limit of quantitation; HCA: hierarchical clustering analysis; PCA: Principal components analysis; PC: Principal component; PLS-DA: Partial least squares discriminant analysis; VIP: variable importance of projection.

\section{Declarations}

\section{Acknowledgements}

Not applicable.

\section{Authors' contributions}

Conceptualization, L.Z., Y.L. (Yong-Jun Li), and Y.H.; methodology, C.W., Z.Z., and L.Z.; software, Y.L. (YueTing Li) and Z.G.; investigation, C.W. and Z.Z.; validation, C.W., L.Z., and Y.H.; formal analysis, Z.Z., C.W., and Y.H.; resources, Y.W., A.W., S.C., X.M.; writing-original draft preparation, L.Z. and Z.Z.; writing-review and editing, Y.H. and Y.L. (Yong-Jun Li). All authors have read and agreed to the published version of the manuscript.

\section{Funding}

This research was funded by National key R\&D program of China, grant number 2018YFC1708100; National natural science foundation of China, grant number 81960763/U1812403; Guizhou science and technology 
department, grant number [2019] 5407/5660, [2018]4006, [2017]5601; Guiyang science and technology bureau, grant number [2017]30-29.

\section{Availability of data and materials}

The research data generated from this study is included within the article.

\section{Ethics approval and consent to participate}

Not applicable.

\section{Consent for publication}

Not applicable.

\section{Competing interests}

The authors declare that they have no competing interests.

\section{Author details}

${ }^{1}$ State Key Laboratory of Functions and Applications of Medicinal Plants, Guizhou Provincial Key Laboratory of Pharmaceutics, Guizhou Medical University, 4 Beijing Road, Guiyang 550004, China;

${ }^{2}$ Engineering Research Center for the Development and Application of Ethnic Medicine and TCM, Ministry of Education, 4 Beijing Road, Guiyang 550004, China; ${ }^{3}$ School of Pharmacy, Guizhou Medical University, 4 Beijing Road, Guiyang 550004, China.

\section{References}

1. Qiu D, Du J. Chinese Materia Medica · Miao Yao. Guiyang: Guiyang Science and Technology Press; 2005.

2. Commission CP. Pharmacopoeia of the People's Republic of China: Part One. Beijing: China Medical Science Press; 2015. p. 20.

3. Li Y, Xiong C, Zeng D, Wang Y, Cheng J, Wu G, et al. Investigation and Identification of Individual Varieties of Miao Medicine Xueteng. Journal of Medicine and Pharmacy of Chinese Minorities. 2004(S1):149-51.

4. Lu Y, Chen H, Lu J. Study on the Quality Standard for Guyanning Granules. Lishizhen Medicine Materia Medica Research. 2007;18(10):2483-5.

5. Xiong Y, Cheng Z, Peng C, Wang Z. Miao Medicine Shengxian Soup and Herbal Fumigation Unite Treatment Knee Osteoarthritis 120 Cases Clinical Curative Effects Observation. Liaoning Journal of Traditional Chinese Medicine. 2010;37(12):2397-9.

6. Tang C, Zhu G, Cai J, Cao P. The Influence of Tongqiao Huashuan Decoction of Miao medicine on VEGF Expression of cerebral infarction area in acute stage of MCAO Rats. Henan Traditional Chinese 
Medicine. 2016;36(4):596-9.

7. G R. R M, S S-KJ. Triterpene saponins from the Chinese drug "Daxueteng" (Caulis sargentodoxae). Planta Med. 1991;57(5):468-70.

8. Wang Y, Li H, Zhou J, Du F. Synergistic Antibacterial Effect of Effective Components of S. Cuneataã Sufnuticosa and P. Lactiflora for Pathogenic Enteral Bacteria. Chinese Journal of Surgery of Integrated Traditonal Western Medicine. 2008;14(4):410-3.

9. Wang Y, Li H, Zhou J, Du F, Luo D, Wu X. Determination of the effective fractions in the Daxueteng and the study of its inhibitory activity against the bacterium in the abdominal cavity. Chinese Traditional Patent Medicine. 2008;30(8):1230-2.

10. Wang K, Li S, Liang B. Research on the Effect of Compound Daxueteng on Chronic Nonbacterial Prostatitis in Rats. Journal of Guiyang Medical College. 2009;34(3):304-7.

11. Li H, Huang S, Deng C, Zhang H. Study on Analgesic and Anti-inflammatory Effects of Daxueteng. Shaanxi Journal of Traditional Chinese Medicine. 2013;34(10):1427-8.

12. Administration GMP. Guizhou Province Chinese herbal medicines, national herbal medicines quality standards. Guiyang: Guizhou Science and Technology Publishing House; 2003. p. 431.

13. Li W, Gui S, Wang J, Tao Y, Shi L. Study on Fingerprint of Sargentodoxa cuneata from Different Habitats and Determination of Salidroside and Chlorogenic Acid as Two Components. Journal of Anhui Traditional Chinese Medical College. 2014;33(6):82-6.

14. Wang W, Zhang Z, Tang H, Lin H, Sun Y, editors. Determination of Salidroside in Sargentodoxa cuneata by HPLC. The 7th Chinese Medicine Analysis Academic Exchange Conference; 2014; Guangzhou.

15. Wang Y, Hong W, Wang J. Simultaneous determination of protocatechuic acid and chlorogenic acid in Daxueteng by HPLC. New Journal of Traditional Chinese Medicine. 2016;48(2):241-3.

16. Ge M, Li J, Zhang L, Jin Z, Zhong Z. HPLC Analysis to Flavonoid Compound of Sargentodoxa Cuneata Leaves. Journal of Zhejiang College of Traditional Chinese Medicine. 2002;26(6):71-2.

17. Wang W, Zou J, Huang B, Zhao X, Hong Y, Zhang G. Colorimetric determination of total flavonoids in Daxueteng and its removal of DPPH free radicals. Jiangsu Agricultural Sciences. 2014;42(11):356-8.

18. Liu J, Zhang Z, Li X, Wu X, Zhang Y, Shu Z. Purification of the proantho cyanidins from Sargentodoxa cuneata (Olive.) Rehd.Et Wils.by macroporous resin. Science Technology of Food Industry. 2017;38(1):112-5.

19. Gao Y, Zhao D, Liu J, Huo X. Studies on the chemical constituents of the volatile oil from Caulis sargentodoxae. Chinese Traditional Patent Medicine. 2004;26(10):843-5.

20. Ma B, Gao Z. Quantitative determination of emodin in the vine of Sargentodoxa cuneata by RP-HPLC. Journal of Beijing University of Traditional Chinese Medicine. 2005;28(1):57-9.

21. Li H, Zhao F, Yuan X, Liu X, Chen L, Yan L, et al. Determination of phenols and triterpenoid saponins in stems of Sargentodoxa cuneata. China Journal of Chinese Materia Medica. 2015;40(10):1865-71.

22. Li D-H, Lv Y-S, Liu J-H, Yang L, Wang Y, Zhang S-K, et al. Simultaneous Determination of Four Active Ingredients in Sargentodoxa cuneata by HPLC Coupled with Evaporative Light Scattering Detection. International journal of analytical chemistry. 2016;2016. 
23. Aissi O, Boussaid M, Messaoud C. Essential oil composition in natural populations of Pistacia lentiscus L. from Tunisia: Effect of ecological factors and incidence on antioxidant and antiacetylcholinesterase activities. Industrial Crops Products. 2016;91:56-65.

\section{Table 4}

Table 4 The content of nine components in thirty batches of DXT $(\mathrm{mg} / \mathrm{g}, n=3)$ 


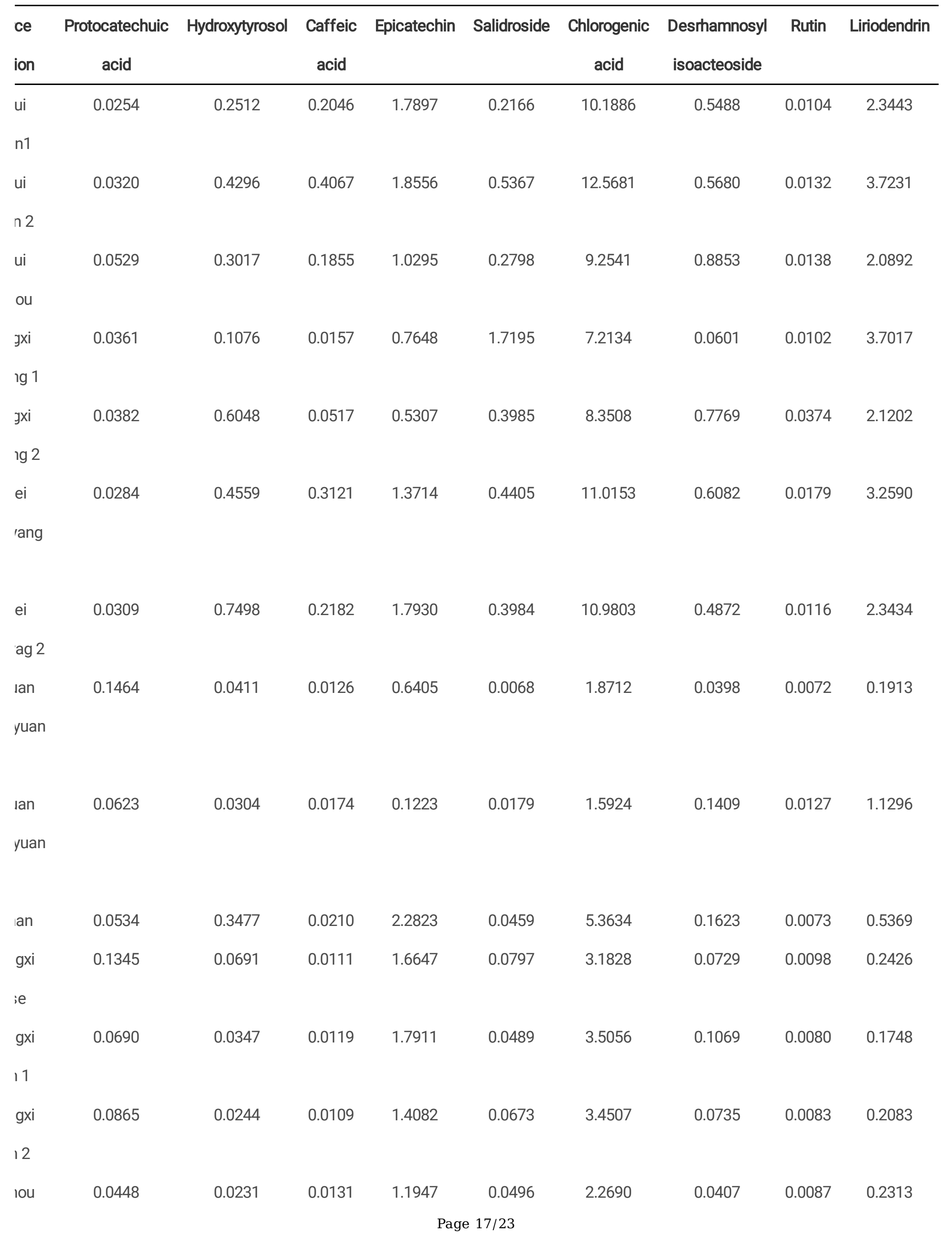


$\lg 1$

Iou

0.1657

0.0398

0.1299

0.1344

0.0336

0.9705

0.0966

0.0083

1.7408

$\lg 2$

Iou

0.1184

0.0289

$0.0107 \quad 0.5208$

0.0368

1.3388

$0.0348 \quad 0.0079$

0.1226

$\lg 3$

iou

0.0412

$0.1665 \quad 0.0342$

0.1270

0.3221

2.9189

0.0620

0.0129

3.3263

$\lg 4$

Iou

0.0509

0.0314

0.0119

1.0830

0.0701

3.6517

$0.0508 \quad 0.0099$

0.1294

n 1

Iou

0.0373

0.8521

0.0453

1.0473

0.5242

6.1858

0.1673

0.0271

2.3480

n 2

Iou

0.0406

0.2006

0.0221

0.5140

0.4027

3.7482

0.7132

0.0229

2.4964

n 3

Iou

0.0424

0.3792

$0.0362 \quad 1.0309$

0.2606

5.6633

0.5337

$0.0212 \quad 1.8998$

n 4

IOU

0.0673

0.0793

0.0119

1.8180

0.1286

2.7996

0.0415

0.0097

0.2659

n 5

Iou

0.0398

0.0646

0.0226

0.4699

0.1047

2.2999

0.6492

0.0198

1.2611

1

Iou

0.0938

0.1820

0.0467

0.8132

0.1388

2.3836

0.0250

0.0170

1.8512

2

Iou

0.1106

0.0158

1.0108

0.3721

0.0027

0.2401

0.0354

2.0657

2.0657

3

Iou

0.0504

0.0795

$0.0172 \quad 1.7105$

0.1191

4.4098

0.1029

0.0086

0.4374

shui

Iou

0.0254

0.2263

0.0150

1.8509

0.1061

4.7889

0.1329

0.0102

0.1373

shui

10u

0.0541

$0.1057 \quad 0.0161$

1.1253

0.0542

2.0905

0.1138

0.0088

0.2770 
un

IOU

0.0348

0.6470

0.0233

\section{Figures}

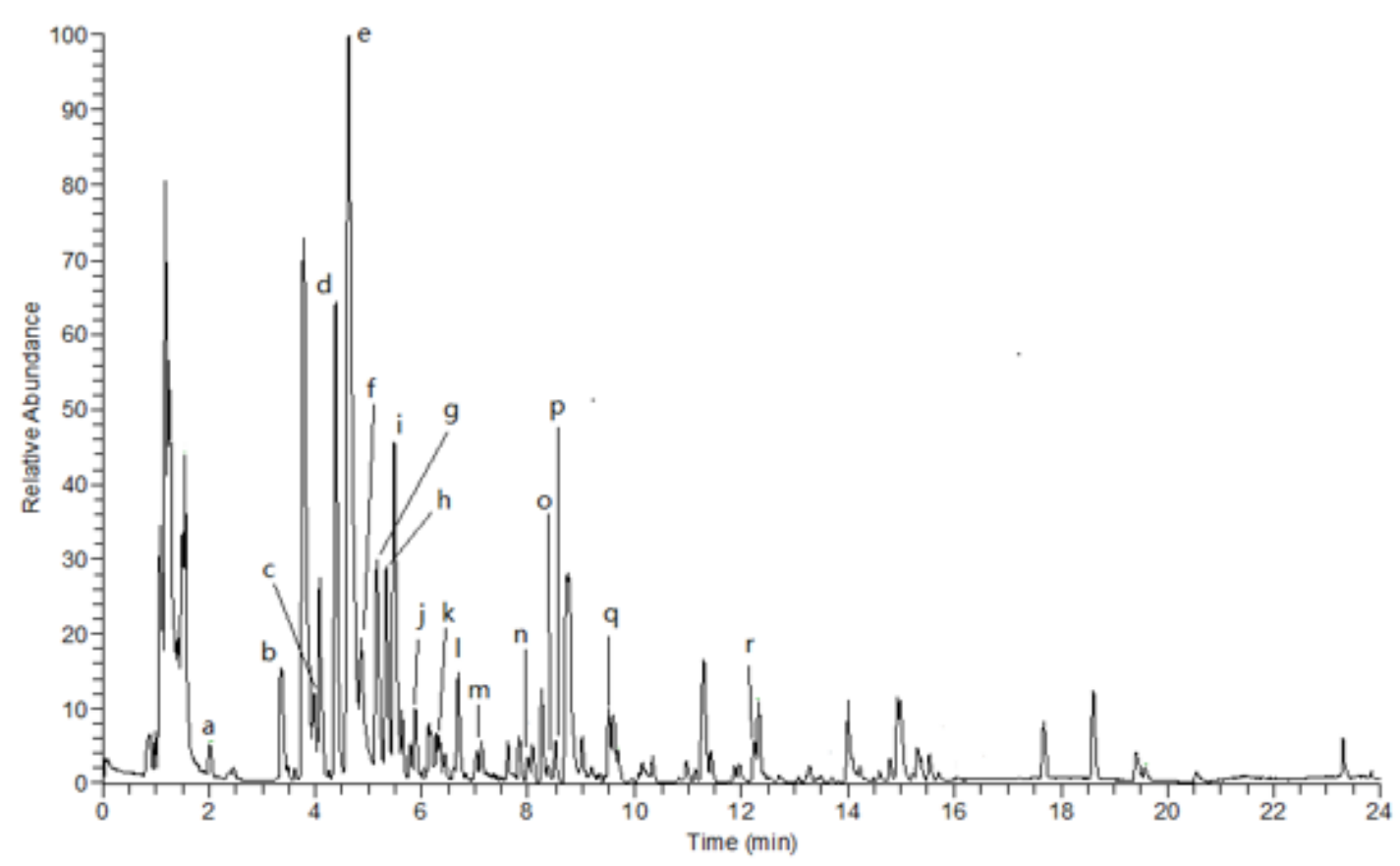

\section{Figure 1}

Total ion chromatogram of DXT in negative mode (a) Vanillic acid; (b) Protocatechuic acid; (c) Hydroxytyrosol; (d) Salidroside; (e) Chlorogenic acid; (f) p-hydroxybenzoic acid; (g) Caffeic acid; (h) Epicatechin; (i) Desrhamnosyl isoacteoside; (j) Ferulic acid; (k) Feruloyltyramine; (l) Madasiatic acid; (m) Procyanidin B2; (n) Liriodendrin; (o) Physcion; (p) Rutin; (q) Rosamultin; (r) Oleanolic acid 


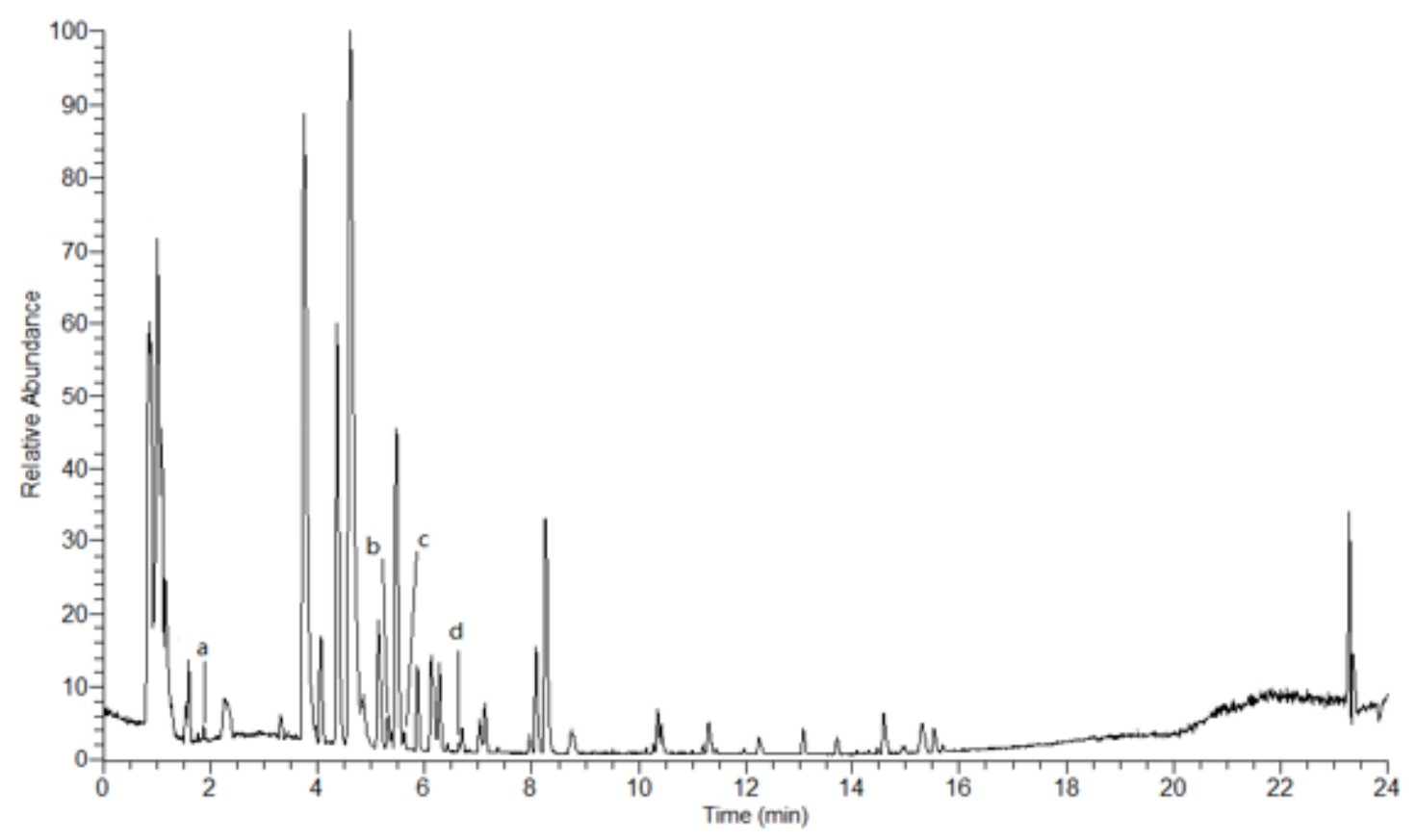

Figure 2

Total ion chromatogram of DXT in positive mode (a) Apocynin; (b) Epicatechin; (c) Emodin; (d) Quercetin
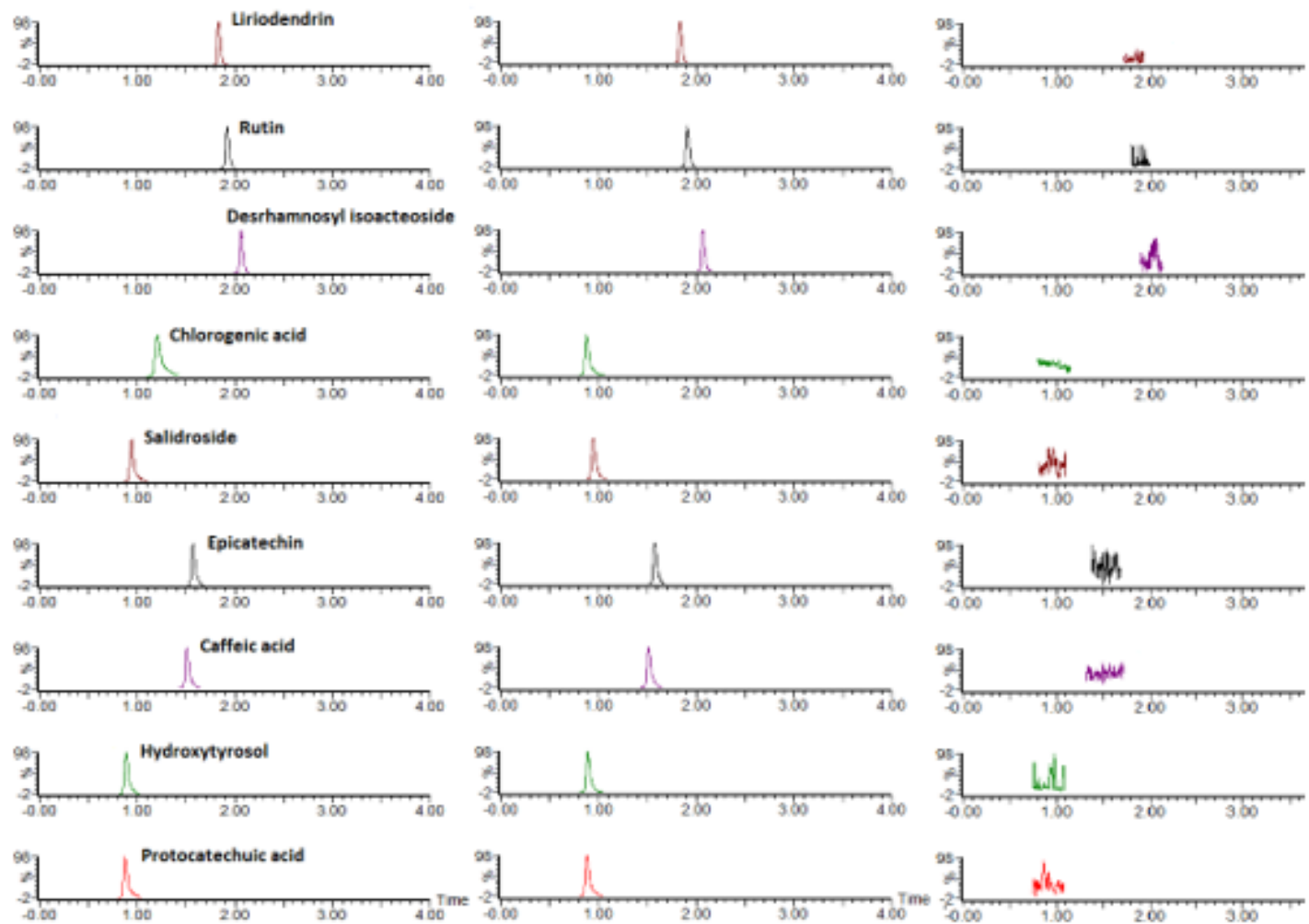

(a)

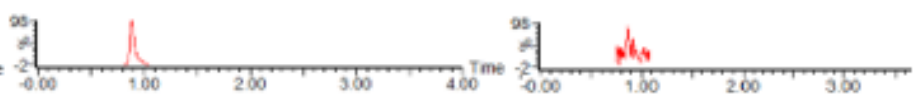

(b)

(c)

\section{Figure 3}

The extracted ion chromatogram of nine components: (a) mix standards; (b) samples; (c) blank solvents 


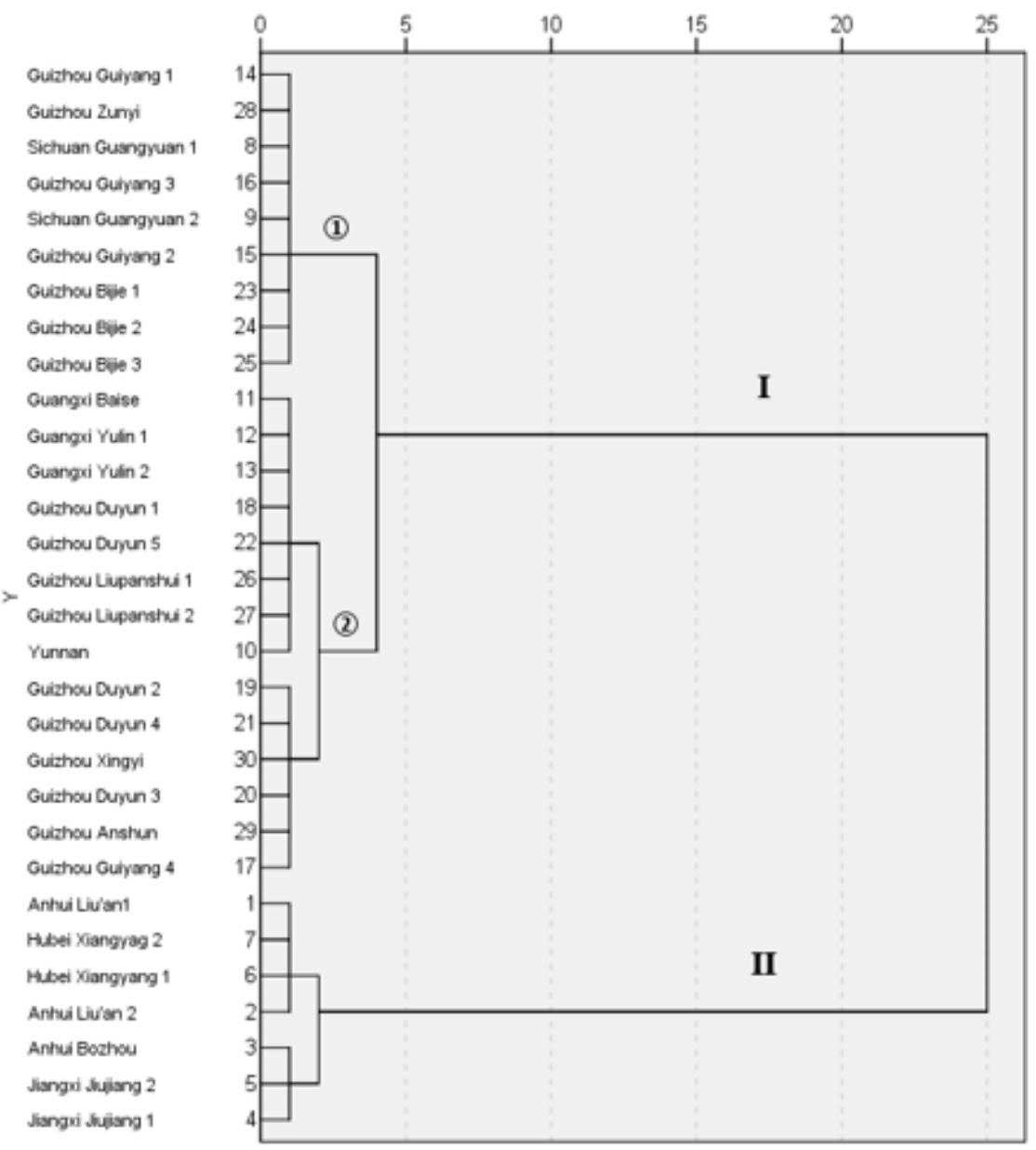

Figure 4

HCA dendrogram 


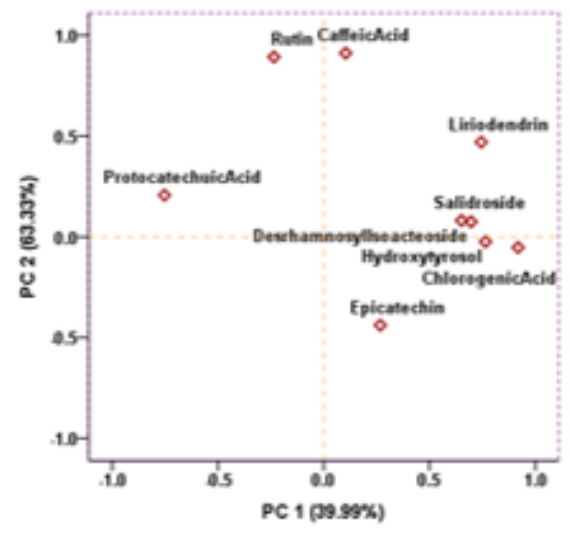

(a)

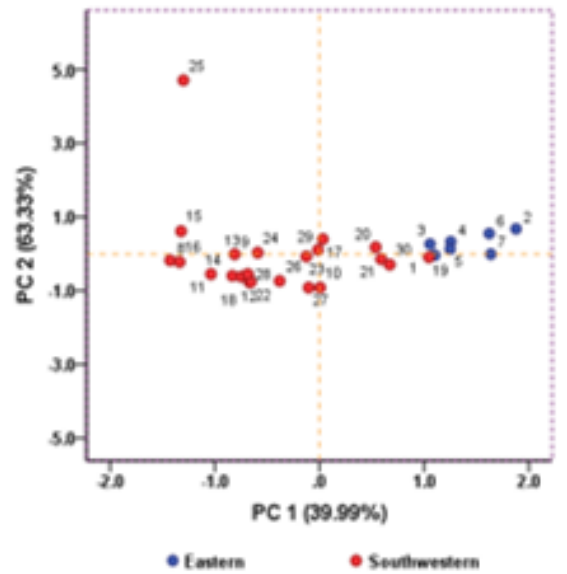

(c)

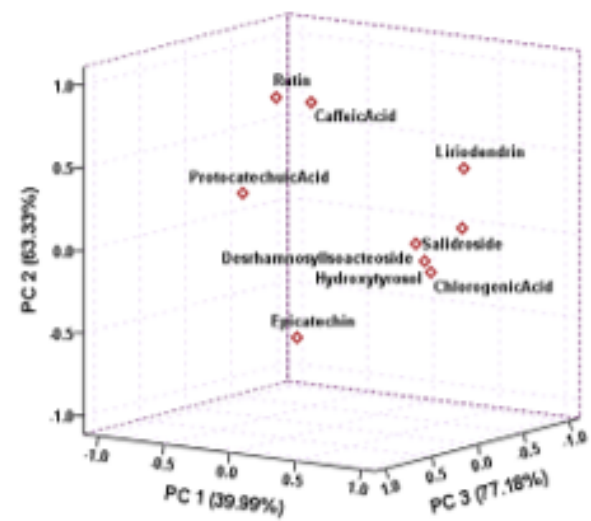

(b)

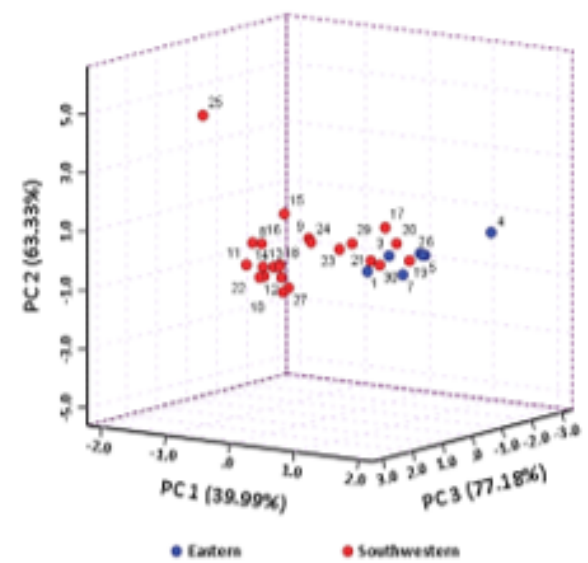

(d)

\section{Figure 5}

PCA plot of DXT (a) 2D loading plot; (b) 3D loading; (c) 2D score plot; (d) 3D score plot

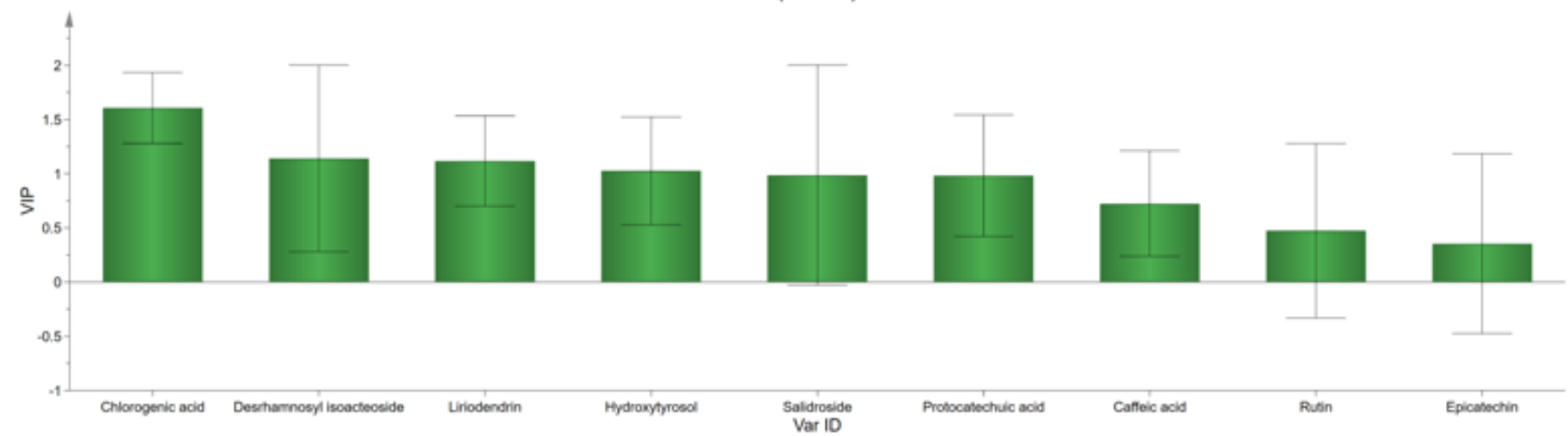

Figure 6 
VIP value obtained from PLS-DA

\section{Supplementary Files}

This is a list of supplementary files associated with this preprint. Click to download.

- additonalfile1.docx

- additonalfile1.docx

- additionalfile2.docx

- additionalfile2.docx

- Additionalfile3.docx

- Additionalfile3.docx

- Additionalfile4.docx

- Additionalfile4.docx 